\title{
氮化硼纳米管热输运性能的分子动力学模拟
}

\author{
高宇飞 ${ }^{1}$ 孟庆元 ${ }^{1}$ 张 璐 $^{2}$ 刘甲秋 ${ }^{3}$ 荆宇航 ${ }^{1, *}$ \\ ('哈尔滨工业大学航天学院, 哈尔滨 150001; 2中国医科大学, 沈阳 110001; 3哈尔滨玻璃钢研究院, 哈尔滨 150001)
}

\begin{abstract}
摘要: 采用基于声子散射理论的 Boltzmann-Peierls 声子传输方程(BTE)和非平衡态分子动力学模拟(NEMD) 方法研究了氮化硼纳米管(BNNT)的热输运性能. 分析了 BNNT 的热力耦合效应, 通过 BTE与 NEMD两种方法 相结合, 分析了温度和长度对 BNNT 热输运性能的影响, 并应用量子修正扩大了 NEMD 的研究范围. 结果表 明: 随着拉伸或压缩应变的增加, BNNT 热输运性能均呈降低的趋势. 通过计算声子态密度(PDOS)在理论上分 析了以上结果, 发现在拉伸状态下, 声子模式的变化是决定 BNNT 热输运性能变化的主要因素; 在压缩状态下, 热导率变化是由于模型发生明显的屈曲变形引起的. 在低温段, BNNT 的热输运性能受量子效应影响最初有一 个线性增加的过程, 当温度超过一定值时, 其开始显著地降低; 当BNNT 长度小于 $120 \mathrm{~nm}$ 时, 随着长度的增 加, 其弹道性能逐渐减弱, 但仍主要体现为弹道特征, 其热导率 $(K)$ 与长度 $(L)$ 基本满足 $K \propto L^{\alpha}$ 这一关系.
\end{abstract}

关键词：氮化硼纳米管; 热输运性能; Boltzmann-Peierls 声子传输方程; 非平衡态分子动力学模拟;

声子态密度; 量子修正

中图分类号: $0641 ; 0642$

\section{Molecular Dynamics Simulation of Thermal Transport Properties for Boron Nitride Nanotubes}

\author{
GAO Yu-Fei ${ }^{1} \quad$ MENG Qing-Yuan ${ }^{1} \quad$ ZHANG Lu² LIU Jia-Qiu ${ }^{3} \quad$ JING Yu-Hang ${ }^{1, *}$ \\ ('School of Astronautics, Harbin Institute of Technology, Harbin 150001, P. R. China; ${ }^{2}$ China Medical University, \\ Shenyang 110001, Liaoning Province, P. R. China; ${ }^{3}$ Harbin FRP Research Institute, Harbin 150001, P. R. China)
}

\begin{abstract}
The Boltzmann-Peierls phonon transport equation (BTE) and non-equilibrium molecular dynamics simulation (NEMD) are used to investigate the thermal transport properties of boron nitride nanotubes (BNNTs). First, the thermal-mechanical coupling is explored using NEMD. Then, by combining BTE and NEMD, the influence of temperature and length is investigated. Quantum correction is used to extend the range over which NEMD can be used. The results demonstrate that under low-strain conditions, the thermal conductivity decreases with increasing tensile or compressive strain. Then the phonon density of state (PDOS) is used to analyze the trends in thermal transport properties theoretically; it is found that the variations in thermal transport properties under tension are caused by changes in the phonon modes, and that under compression changes are induced by the flection of the BNNT structure. The BNNT thermal conductivity increases linearly with increasing temperature because of the quantum effect at low temperatures, and it decreases significantly as the temperature reaches a certain value. When the BNNT length is less than $120 \mathrm{~nm}$, the BNNT's ballistic characteristics weaken with increasing length, but it also performs ballistic characteristic mainly, and thermal conductivity $(\kappa)$ and length $(L)$ obey the relationship $k \propto L^{\alpha}$.
\end{abstract}

Received: November 30, 2011; Revised: January 28, 2012; Published on Web: February 27, 2012.

"Corresponding author. Email: jingyuhang@gmail.com; Tel: +86-451-86414143.

The project was supported by the National Natural Science Foundation of China (10772062).

国家自然科学基金(10772062)资助项目

(C) Editorial office of Acta Physico-Chimica Sinica 
Key Words: Boron nitride nanotubes; Thermal transport property; Phonon Boltzmann-Peierls transport equation; Non-equilibrium molecular dynamics simulation; Phonon density of state; Quantum correction

\section{1 引言}

纳米材料因其独特的结构和优良的力学、电 学、热学、光学等性能, 表现出广阔的应用前景. 至 1991 年发现碳纳米管(CNT) 以来, ${ }^{1}$ 各种不同种类的 纳米管相继被发现, 对它们的研究也随之展开.

1994 年 Rubio 等 ${ }^{2}$ 预测到一定存在氮化嗍纳米 管(BNNT). 之后人们在实验中得到了多壁 BNNT. 1996 年 Loiseau 等 ${ }^{3}$ 采用电弧放电法得到了单壁 BNNT. 有关 BNNT 及氮喼化合物的研究, 如电子结 构和力学性能的研究已经展开, ${ }^{4}$ 但有关其热学性能 的研究却较少, 在力学和热学交叉领域, 如热力耦 合效应方面还未见报道. BNNT 作为一种典型的纳 米材料, 它的很多结构参数都对其热导率, 尤其是 轴向热导率有着显著的影响, ${ }^{5}$ 因此, 可以利用 BNNT 的这种优良特性制成可控制的导热设备, 这 与传统的通过选择不同的材料来实现对器件导热 性能控制的方法相比有了革命性的突破. 因此有必 要对 BNNT 的热传导机理和导热性能进行系统的 研究.

目前针对 BNNT 导热性能的研究成果指出: 对 BNNT 的热输运起主要贡献的是声子, 这与 CNT 的 热输运机理不同(对 CNT 热导率起主要作用的是声 子和电子). BNNT 的热导率低于 CNT, 但 BNNT 比 CNT 有着更强的热稳定性和化学稳定性; Liew 和 Yuan ${ }^{6}$ 通过分析 CNT 和 BNNT 混合物发现, BNNT 具有更好的稳定性, 较之 CNT 更适宜做管状结构 的外壁; 然而也有部分研究 ${ }^{7}$ 指出, 在低温段, BNNT 的热导率要高于 CNT. 此外, 同位素对 BNNT 热导 率有着显著的影响, Zettl等 ${ }^{8,9}$ 的研究指出, 当BNNT 中含有高浓度的 B 同位素时, 其热导率可与 $\mathrm{CNT}$ 相 比; 纯的 ${ }^{11} \mathrm{~B}$ 同位素可将 BNNT 热导率提升 $50 \% ;{ }^{10}$ 而 Barman ${ }^{11}$ 的研究表明, 同位素 ${ }^{10} \mathrm{~B}$ 会削弱氮化嗍晶 体的热导率. BNNT 具有较好的导热性能, 在室温 下, 外直径为 30-40 nm 的 BNNT 的热导率可达到 $350 \mathrm{~W} \cdot \mathrm{m}^{-1} \cdot \mathrm{K}^{-1}$; Stewart 等 ${ }^{12}$ 通过计算表明 $(8,0)$ BNNT 的热导率为 $200 \mathrm{~W} \cdot \mathrm{m}^{-1} \cdot \mathrm{K}^{-1}$. Nakayama 等 ${ }^{13}$ 发 现氮化硼纳米带可以增强部分金属氧化物的导热 性能. Mingo 和 Broido ${ }^{14}$ 通过研究输运方程和弹道函 数发现纳米管的热传导性能表现出弹道特征. 以上
结果表明: BNNT 在扫描探针显微镜、原子力显微镜 的针尖, 高温陶瓷以及纳米尺度热量计和微电子处 理器等方面有着很大的潜在应用. 但由于实验测量 中的实际困难和材料在纳米尺度下的误差, 目前理 论和实验针对纳米管导热性能的定量研究结果具 有较大的差别, 如有部分研究结果 ${ }^{15,16}$ 表明 BNNT 具 有极低的热导率( 热导率约 $1.5-14 \mathrm{~W} \cdot \mathrm{m}^{-1} \cdot \mathrm{K}^{-1}$ ). 总 体来说, 目前对于 BNNT 热输运机理和性能的定量 研究成果仍很缺乏, 而且随着声子散射理论的逐步 完善, 一些对 BNNT 导热性质产生重要影响的较复 杂的因素, 如纳米管的弹道传输与扩散传输的分界 性能、应变作用下动态纳米管结构的热输运性能等 逐渐被提出, 并逐渐成为研究的热点.

本文首先采用非平衡态分子动力学模拟 (NEMD)方法研究了 BNNT 在轴向应变作用下的热 输运性能, 并通过计算声子态密度(PDOS)的方法对 模拟结果进行了理论上的补充和验证. 进一步通过 求解 Boltzmann-Peierls 声子传输方程 (BTE) 和 NEMD 相结合的方法分析了温度和长度对 BNNT 热输运性能的影响, 通过量子修正扩大了 NEMD的 研究范围. 为研究 BNNT 弹道传输与扩散传输的分 界以及动态纳米管结构的热输运性能提供了一些 理论与计算模拟的参考数据.

\section{2 计算模型与方法}

\section{1 非平衡态分子动力学模拟方法}

本文在模拟过程中应用 Tersoff多体势 ${ }^{17}$ 来描述 BNNT 中原子间的相互作用. 求解原子的运动方程 时采用预测一校正算法, 积分步长为 $0.5 \mathrm{fs}$. 边界条 件选择为自由边界条件. 构建 BNNT 模型时, 首先 根据 BNNT 的空间结构特点得到其初始构型的空 间排列, 然后将 BNNT 的初始构型在 $0 \mathrm{~K}$ 下弛豫 50000 步, 随后逐渐升温, 每次升温 $100 \mathrm{~K}$, 并驰豫相 同的步数, 直到温度升到 $1600 \mathrm{~K}$, 最后进行退火处 理来得到其稳定结构.

计算模型的热导率采用 NEMD, ${ }^{18}$ 其基本思想 为: 将 BNNT 沿轴向等分为 $N$ 层, 设 0 层和 $N / 2$ 层为 “冷区”和“热区” (见图 1). 每隔一定步数提取 0 层中 动能最大的原子和 $N / 2$ 层中动能最小的原子, 并交 


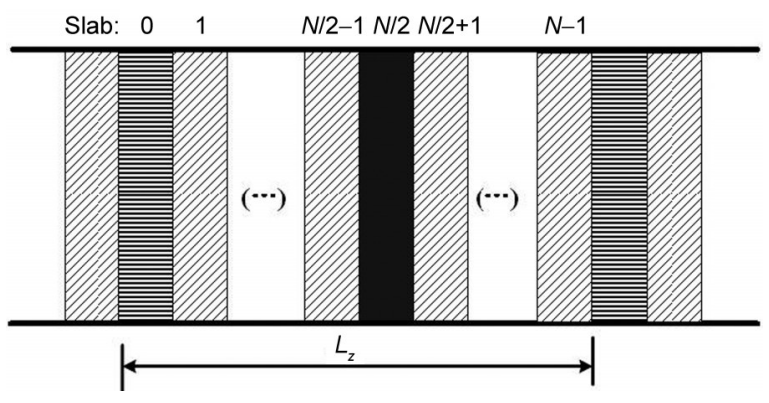

图 1 非平衡态分子动力学模拟过程示意图

Fig.1 Schematic diagram of NEMD process

换两者速度. 一般情况下冷区中动能最大的原子的 动能大于热区中动能最小的原子的动能, 这样便产 生了一个从 0 层到 $N / 2$ 层的热流. 又因为热区的温度 高于冷区的温度, 因此便存在一个与热流方向相反 的温差. 基于上述模型, 经过统计可获得轴向各层 的温度, 进一步计算可得温度梯度 $(\nabla T)$, 再结合能 量交换过程中统计得到的热流密度 $(J)$, 由 Fourier 导 热定律 $J=-\kappa \nabla T$, 可计算得到 BNNT 的热导率 $(\kappa)$. 整个非平衡态分子动力学模拟过程是通过 Lammps 开源软件包 ${ }^{19}$ 实现的.

图 2 为 $\mathrm{BNNT}$ 在 $300 \mathrm{~K}$ 温度下轴向方向的温度 分布图, 其中各层温度是通过统计各层中所有原子 的动能, 通过公式(1)获得

$$
\sum_{i} \frac{1}{2} m_{i} v_{i}^{2}=\frac{3}{2} N k_{\mathrm{B}} T
$$

其中 $N$ 为层中的原子数, $T$ 代表温度, $k_{\mathrm{B}}$ 为 Boltzmann 常量, $m_{i}$ 和 $v_{i}$ 分别为模型中 $i$ 原子的质量和速度. 从 图中可发现中间各层的温度呈线性分布, 而在冷层 和热层附近区域, 则有明显的非线性温度响应, 这 是由于热源和热槽的强相互作用产生的. ${ }^{20}$ 因此在 计算BNNT 热导率过程中, 需要统计中间各层的温

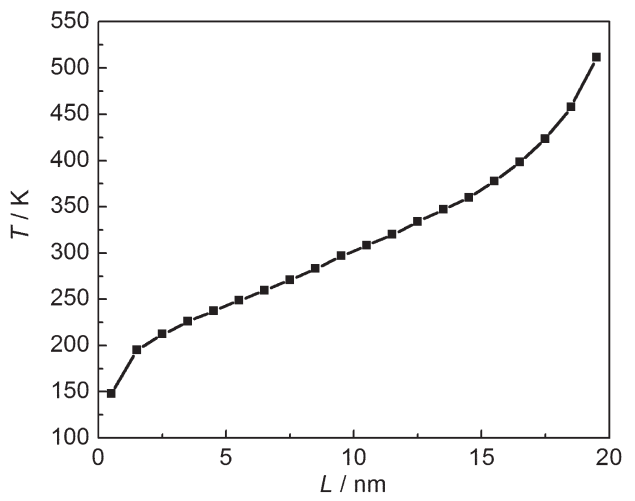

图 2 BNNT 轴向 $(L)$ 的温度分布

Fig.2 Temperature distribution of BNNT along axial direction $(L)$
度, 进而获得 BNNT 轴向方向的温度梯度以及热导 率.

模拟的整个过程分为五步: 首先用 Conjugate Gradient $(\mathrm{CG})$ 方法弛豫整个构型, 以获得 BNNT 的 稳定结构; 然后将纳米管两端固定, 在 NPT 系综下 运行 10000 步(5 ps)标定压力使其处于最小状态; 再 在最小压力状态下运行 100000 步(50 ps), 使系统达 到稳定状态; 然后在 NVT 系综下运行 200000 步 (100 ps)来弛豫整个系统; 最后在 NVT 系综下运行 1000000 步 $(500 \mathrm{ps})$ 统计热流密度和温度梯度, 计算 得出 BNNT 的热导率(在最后一步过程中每 100000 步统计一次热导率, 通过计算 10 个热导率数值的偏 差分布可获得模型热导率的不确定度).

\subsection{Boltzmann-Peierls 声子传输方程方法}

微观固体中的热量主要是靠晶格振动产生的 声子传输的, 声子是一些被抽象出的离散粒子, 单 一声子的能量为 $\hbar \omega, \hbar=h / 2 \pi, h$ 为普朗克常量, $\omega$ 为 频率. Boltzmann-Peierls 声子传输方程 ${ }^{14,21}$ (BTE) 可以 完整地描述固体中声子间的相互作用. 但获得 BTE 的精确解是困难的, 目前可以通过一些近似的方法 来获得不同模型对应的 BTE 的近似解. 求解 BTE 的 基本思想是通过不同声子的散射时间和处于非平 衡态声子的分布, 并结合模型的边界条件来获得模 型热流与温度梯度, 最后求得模型的热导率.

\section{3 低温段量子修正}

一般情况下, 经典分子动力学模拟(MD)方法在 温度接近和高于德拜温度时被认为是有效的, 因为 在此温度区域内所有的声子模式都被完全激发出 来. 与此相对的, 当温度显著地低于德拜温度时, 大 量的声子没有被激发, 模型的量子效应不能被忽 略, 此时经典的 MD 方法无效. 为突破这一限制, 量 子修正方法 $(\mathrm{QCs})^{22-25}$ 被提出, 目前它被广泛应用于 热力学和力学的研究中.

QCs 的基本思想是由经典力学系统中的模拟温 度来计算量子系统中对应的修正温度, 根据经典力 学系统与量子系统能量相等, 具体为系统的动能等 于声子能量的一半这一原则. 模拟温度 $\left(T_{\mathrm{MD}}\right)$ 与修正 温度的关系可表示为

$$
3 N k_{\mathrm{B}} T_{\mathrm{MD}}=\int_{0}^{\infty} g(\omega) N_{0}\left(\omega, T_{\mathrm{q}}\right) \hbar \omega \mathrm{d} \omega
$$

其中, $N_{0}$ 为平衡态声子分布函数, $T_{\mathrm{q}}$ 为量子修正温 度, $g(\omega)$ 为声子态密度. 结合模拟中获得的 $T_{\mathrm{MD}}$ 可计 算 $T_{\mathrm{q}}$. 通过统计不同时间的轴向热流密度和温度梯 
度, 可获得修正后的热导率.

除了基于 $\mathrm{MD}$ 的 $\mathrm{QCs}$ 修正外, 还存在两种基于 势函数的量子修正方法, Feynman-Hibbs 势函数( $\mathrm{FH}$ 势)和 Wigner-Kirkwood 势函数(WK 势)方法. FH 势 是由量子均分方程的 Feynman-Hibbs 估算变量获得 的, 是一种依赖于温度的二体势, 可以方便地植入 分子动力学或蒙特卡罗算法的计算程序中. 应用这 种方法, Sese $^{26}$ 计算了 Lennard-Jones 系统的热力学 和结构特性, Tchouar 等 ${ }^{27}$ 应用由 FH 方法演化的 Lennard-Jones 势研究了氛的热力学、结构和传输特 性, Goharshadi 等 ${ }^{28}$ 应用 Hartree-Fock dispersion 近似 势函数和 $\mathrm{FH}$ 势研究了氦的热力学性质. WK势是由 对均分方程中德布罗意波长的扩展获得的, 可以被 用来研究不同物理特性系统的量子效应.

\section{3 计算结果与讨论}

\section{1 热力耦合效应}

本文选取 $20 \mathrm{~nm}$ 的 20_20扶手椅型 BNNT(原子 数为 6320 个)为研究对象, 分别对模型施加在 $0-0.1$ 范围的轴向拉伸和压缩应变. 施加位移载荷过程 中, 应变是通过重新标定模型轴向原子坐标实现 的, 具体为首先选择 BNNT 轴向方向最外部两层原 子, 分别对其施加反方向的轴向位移载荷, 每施加 完一次位移载荷让系统驰豫一定步数, 使系统达到 平衡状态. 加载速率为每 5000 个步长施加 0.0025 $\mathrm{nm}$ 的位移. 在此期间统计不同时间 BNNT 轴向方向 的热流密度和温度梯度, 最终计算得到热导率随应 变变化趋势如图 3 所示. 由图 3 可发现, 在拉伸和压 缩应变作用下, BNNT 的轴向热导率随着应变的增 加而逐渐减小.

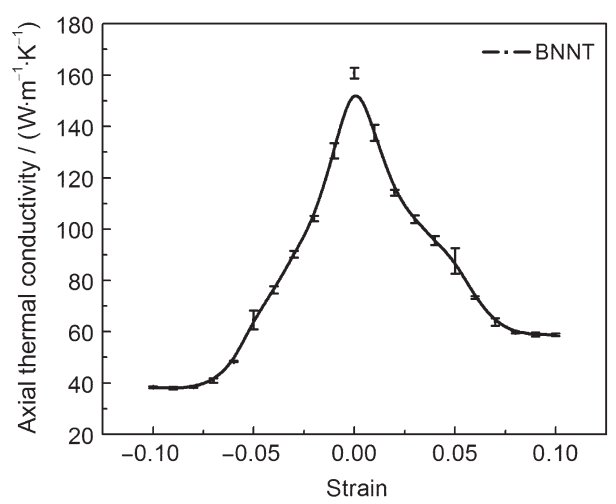

图 3 BNNT轴向热输运性能随轴向应变的变化曲线

Fig.3 Variation curve of axial thermal conductivity of BNNT under axial strain

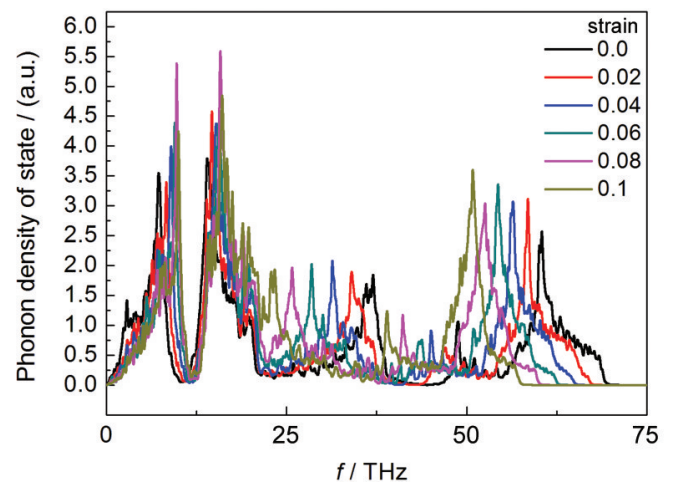

图 4 BNNT 在轴向拉伸应变作用下的声子态密度图

Fig.4 PDOS of BNNT under axial tensile strain

声子态密度(PDOS)可以完整地描述各种模式 声子的分布, 可用于研究动态结构材料的热输运性 能. PDOS 是通过对速度自相关函数进行傅里叶变 换得到的. ${ }^{29-32}$ 速度自相关函数可以表示为

$$
C(t)=\frac{\langle v(t) \cdot v(0)\rangle}{\left\langle v^{2}(0)\right\rangle}
$$

其中

$$
\langle v(t) \cdot v(0)\rangle=\lim _{\tau \rightarrow \infty} \frac{\int_{-\tau}^{\tau} v\left(t+t^{\prime}\right) v\left(t^{\prime}\right) \mathrm{d} t^{\prime}}{\int_{-\tau}^{\tau} \mathrm{d} t^{\prime}}
$$

对(4)式进行傅里叶变换可得到PDOS 的表达形式为

$$
C_{v v}(\omega)=\sum_{k=x, y, z} \sum_{j=1}^{N} \int_{0}^{\infty} \mathrm{e}^{i \omega t} \frac{\left\langle v_{j}^{k}(t) \cdot v_{j}^{k}(0)\right\rangle}{\left\langle v_{j}^{k}(0) \cdot v_{j}^{k}(0)\right\rangle} \mathrm{d} t
$$

其中 $t$ 代表时间, $k=x, y, z$ 代表模型的三个方向. 通过 上式, 结合 $\mathrm{MD}$ 中统计的原子速度, 可以获得不同 应变作用下 BNNT 的声子态密度分布图. BNNT 在 拉伸和压缩应变作用下的声子态密度分布如图 4、

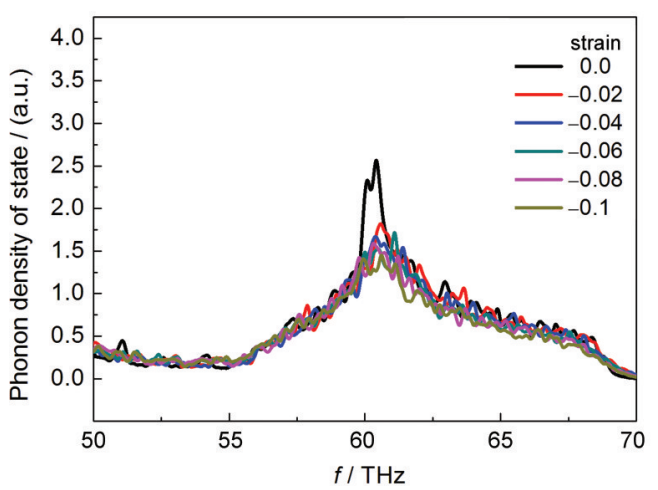

图 5 BNNT 在轴向压缩应变作用下的声子态密度高 频波峰图

Fig.5 High frequency peak in PDOS of BNNT under axial compressive strain 
5 所示.

由图 4 可见, PDOS 中有三个较高的波峰, 其中 前两个位于低频段, 可被视为代表低频声子模式的 波峰, 后一个位于高频段, 被认为是代表高频声子 模式的波峰. 随着应变的变化, 相比于高频波峰, 两 个低频波峰的变化较小. 这意味着高频声子对应变 更加敏感. 由于波峰的变化直接反映了声子数量的 增加或减少, 以及振动模式的增强或减弱, 对比图 4 和图 3 我们可以发现, 高频波峰的左移趋势伴随着 模型热导率的下降, 因此可以认为高频声子对模型 热输运性能有着主要影响.

在 0-0.1 的拉伸应变作用下, 随着拉伸应变的 增加, 从图 4 可以看出高频声子波峰逐渐左移. 这意 味着高频声子数量逐渐减少, 部分高频声子逐渐转 化为低频声子, 它将导致声子的平均频率降低, 因 此此过程可以视为一个红移过程. Xu 和 Buehler ${ }^{33}$ 研 究CNT 在轴向应变作用下的热输运性能, 发现红移 过程将会减小声子的速度. 在声子的弹道输运过程 中, 模型的热导率 $(\kappa)$ 与声子速度有以下关系

$$
\kappa=\frac{1}{3} c l v
$$

其中 $c$ 为比热, $l$ 为平均自由程, $v$ 为声子速度. 因此 图 4 中高频声子的变化趋势将导致 BNNT 的热导率 随着拉伸应变的增加而逐渐减小. 观察图 5 可以发 现, 在压缩应变作用下, 不同应变的 PDOS 变化较 小, 几乎没有明显的变化. 结合图 6 中 0.1 的拉伸和 压缩应变作用下 BNNT 的构型图可以发现, 在 0.1 的拉伸应变作用下 BNNT 被逐渐拉长, 但其没有显 著的塑性变形, 而在 0.1 的压缩应变作用下, BNNT 发生了严重的塑性变形. 随着明显的塑性变形的产 生, 系统的能量将显著降低, 进而导致模型中声子 平均速度的降低, 根据公式(6), 在压缩应变下, BNNT 的热导率随着应变的增加而逐渐减小. 这也 说明了在压缩应变下 BNNT 的热导率发生变化的 机理不同于拉伸应变下的情况: 在拉伸应变下, 是 声子模式的变化导致了 BNNT 热导率的变化; 在压 缩应变下, 是 BNNT 结构的显著变化导致了其热导 率的变化.

Buehler $^{33}$ 和 Zhang ${ }^{32}$ 等分别研究了 CNT 在应变 作用下导热性能, 本文在拉伸应变作用下得到的 PDOS 与 Buehler 和 Zhang 的结果有着相同的变化趋 势, 高频声子波峰均随着拉伸应变的增加而逐渐左 移. 在压缩应变的作用下, Zhang 计算了 $-0.02-0$ 压

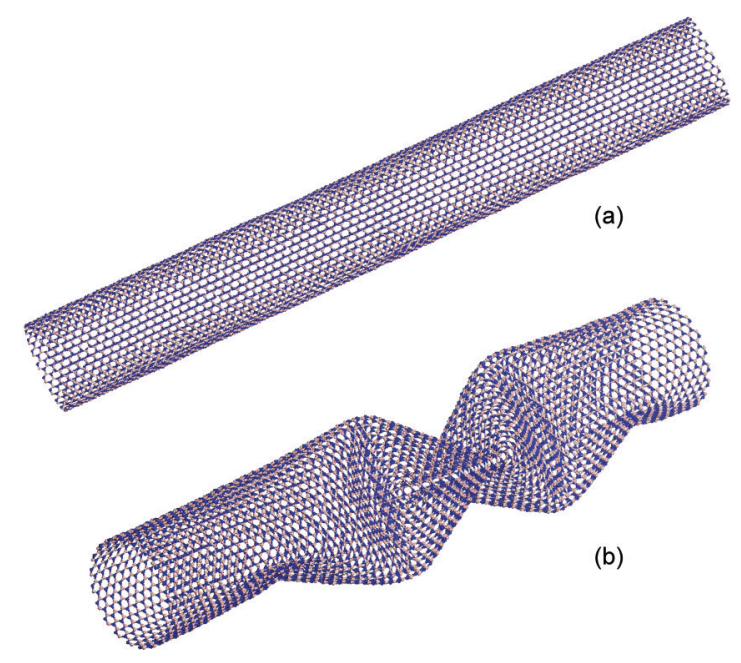

图 6 BNNT 在(a) 0.1 的拉伸应变作用和(b) 0.1 的压缩应变 下的构型图

Fig.6 Structures of BNNT under (a) 0.1 tensile strain and (b) 0.1 compressive strain

缩应变下 CNT 的 PDOS, 发现其高频声子波峰有部 分右移的趋势. 本文认为此处的不同是由于 CNT 的 抗压缩性能强于 BNNT 导致的. 在-0.02 的小压缩 应变作用下 CNT 较之 BNNT 更不容易产生较大的 塑性变形, 因此 CNT 的能量减少将体现在声子模式 的变化上, 而 BNNT 的能量减少体现在结构的变化 上.

\section{2 温度影响}

本文应用 NEMD, 结合低温段温度量子修正方 法研究了 10-1600 K 温度范围内长度为 $20 \mathrm{~nm}$ 的 $10 \_10$ 扶手椅型BNNT(原子数为 3160 个) 的导热性 能. 首先统计模拟过程中 BNNT 的温度 $T_{\mathrm{MD}}$, 结合公 式(2), 可获得量子修正温度 $T_{\mathrm{q}}$. 量子修正温度 $T_{\mathrm{q}}$ 与 模拟温度 $T_{\mathrm{MD}}$ 的关系见图 7. 其中实线为 $T_{\mathrm{q}}$ 随 $T_{\mathrm{MD}}$ 的 变化曲线, 虚线用来对比 $T_{\mathrm{q}}$ 随 $T_{\mathrm{MD}}$ 的变化率, 斜率为 1. 从图中可见在低温段, $\nabla T_{\mathrm{q}} / \nabla T_{\mathrm{MD}}$ 远大于 1 , 随着温 度的升高, 其值逐渐减小, 当温度达到 $1000 \mathrm{~K}$ 时, $\nabla T_{\mathrm{q}} / \nabla T_{\mathrm{MD}} \approx 1$, 这说明此时 $T_{\mathrm{q}}$ 与 $T_{\mathrm{MD}}$ 的变化率一致. 结合 $T_{\mathrm{q}}$, 可获得 BNNT 的热导率随温度的变化趋势 如图 8 所示.

从图 8 可以看出, BNNT 的轴向热导率在低温 段(10-70 K) 随着温度的升高近似呈线性增长趋势; 当温度超过一定值(近似为 $100 \mathrm{~K}$ ) 时, 其热导率随着 温度的升高逐渐降低.

在低温段, 当系统温度远小于德拜温度时, 由 于温度较低, 晶格振动较弱, 被激发出的声子较少. 因此在低温段, 材料的边界对长波声子的散射(也即 


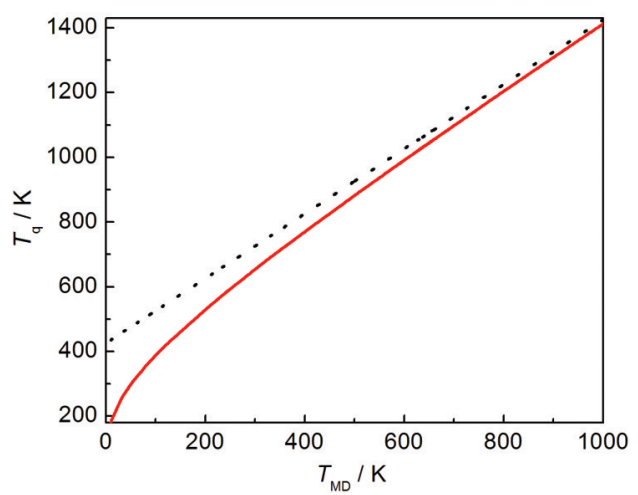

图 7 模拟温度 $\left(T_{\mathrm{MD}}\right)$ 与量子修正温度 $\left(T_{\mathrm{q}}\right)$ 的关系

Fig.7 Relationship between simulated temperature $\left(T_{\mathrm{MD}}\right)$ and quantum corrected temperature $\left(T_{q}\right)$

The slope of dotted line is 1 and can be used to contrast the change rate of corrected temperature with simulated temperature.

边界散射)是影响材料热导率的主要因素. 随着系统 温度的降低, 具有低频率、长波长的声子在声子中 的比重增加. 根据声子理论, 长波声子较易与边界 发生散射, 这就导致了声子平均自由程减小, 由(6) 式可知, 热导率也随之减小, 因此在低温段, BNNT 的热导率随着温度的增加而增加. 在高温段, 晶格 振动剧烈, 散发出大量的声子, 因此声子与声子间 的相互碰撞即声子间的散射取代边界对长波声子 的散射成为影响材料热导率的主要因素. 随着温度 的增高, 声子数增多, 声子间的碰撞概率增大, 声子 平均自由程减小, 因此在高温段, BNNT 的热导率随 着温度的增加而逐渐减小.

由图 8 还可观察到, 在低温段, 修正后的热导率 与分子模拟直接获得的热导率有较大差别, 随着温 度的增加, 这种差距逐渐减小. 文献 ${ }^{23}$ 对比了量子修 正前后硅晶体的热导率变化曲线, 当温度在 $800 \mathrm{~K}$ 左右时, 两者误差约为 $10 \%$. 本文用两种方法获得

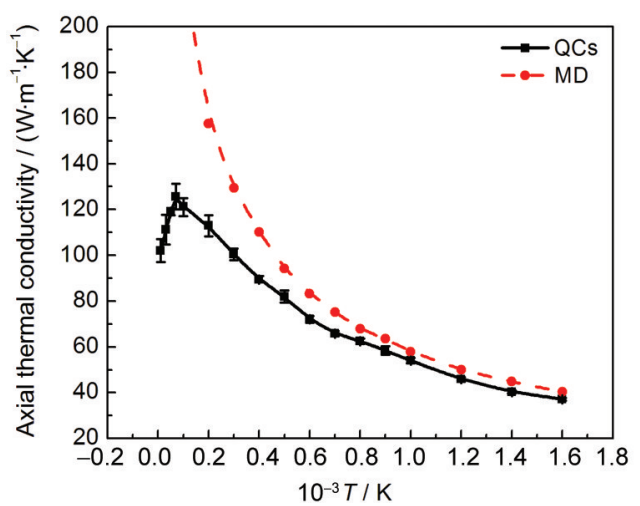

图 8 BNNT 轴向热输运性能随温度的变化曲线

Fig.8 Variation of axial thermal conductivity of BNNT under different temperatures
的数据误差为 $8 \%$. 文献 ${ }^{34}$ 用 MD 计算了 $1200-2000$ $\mathrm{K}$ 下 $(10,10) \mathrm{BNNT}$ 的热导率, 其热导率随着温度升 高逐渐降低, 且 $1200 \mathrm{~K}$ 时 BNNT 热导率近似为 48 $\mathrm{W} \cdot \mathrm{m}^{-1} \cdot \mathrm{K}^{-1}, 1400 \mathrm{~K}$ 时近似为 $43 \mathrm{~W} \cdot \mathrm{m}^{-1} \cdot \mathrm{K}^{-1}, 1600 \mathrm{~K}$ 时近似为 $38 \mathrm{~W} \cdot \mathrm{m}^{-1} \cdot \mathrm{K}^{-1}$. 计算得到上述三个温度的 热导率分别为 $46 、 40 、 37 \mathrm{~W} \cdot \mathrm{m}^{-1} \cdot \mathrm{K}^{-1}$, 与文献 ${ }^{34}$ 结果 较为接近. 文献 ${ }^{35}$ 中通过理论分析 BNNT 的热导率 随温度变化规律, 发现几种直径 $(3,3)(5,0)$ 和几种轴 向长度 $(1,5,10 \mu \mathrm{m})$ 的 BNNT 的热导率发生转折时 系统的温度均在 70-100 K 范围内, 而本文热导率发 生转折的温度在 70-90 K之间, 这可从一个角度印 证本文结果, 可证明本文方法的正确性.

本文通过理论求解 Boltzmann-Peierls 声子传输 方程得出了在低温段 BNNT 的导热性能与外界温 度的关系.

$$
\left.\frac{\partial N}{\partial t}\right|_{\text {drift }}+\left.\frac{\partial N}{\partial t}\right|_{\text {scatt }}=0
$$$$
\text { BTE 声子传输方程 }{ }^{14} \text { 可以表示为 }
$$

左项描述声子漂移部分, 右项描述声子散射部分, 两项可分别表示为 ${ }^{36,37}$

$$
\begin{aligned}
& \left.\frac{\partial N}{\partial t}\right|_{\text {drift }}=-v \nabla T \frac{\partial N_{0}}{\partial T} \\
& \left.\frac{\partial N}{\partial t}\right|_{\text {scatt }}=-n / \tau_{\text {tot }}
\end{aligned}
$$

其中 $N$ 为声子分布函数, $N_{0}$ 为平衡态声子分布函数, $n$ 可表示为 $n=N-N_{0}$, 它用来描述实际声子分布偏离 平衡态的部分, $\tau_{\mathrm{tot}}$ 为总体弛豫时间, 所以(7)式可以 转化为

$$
n=-\tau_{\text {tot }}(v \nabla T) \frac{\partial N_{0}}{\partial T}
$$

结合公式 (10), 热流可表示为

$$
\begin{aligned}
J= & \sum_{s, q} v(s, q) \hbar \omega_{\mathrm{s}}(q) n\left(q, \omega_{\mathrm{s}}\right)= \\
& -\nabla T \sum_{s, q} \tau_{\mathrm{tot}} v^{2}(s, q) \frac{\partial N_{0}\left(\omega_{\mathrm{s}}\right)}{\partial T} \hbar \omega_{\mathrm{s}}(q)
\end{aligned}
$$

其中 $s$ 为声子模式, $q$ 为波数, $\omega$ 为频率. 考虑热流和 温度梯度的关系 ${ }^{38}$

$$
J=-\kappa \cdot V \cdot \nabla T
$$

热导率可表示为

$$
\kappa=\frac{1}{V} \sum_{s, q} \tau_{\mathrm{tot}} v^{2}(s, q) \frac{\partial N_{0}\left(\omega_{\mathrm{s}}\right)}{\partial T} \hbar \omega_{\mathrm{s}}(q)
$$

其中 $V$ 为体积. 在低温段, 影响热导率的主要因素 是声子与边界的散射, 近似认为声子与边界的散射 时间是散射时间 $\tau_{\mathrm{tot}}$ 的主要部分. 边界散射时间可表 
示为

$$
\frac{1}{\tau_{\mathrm{b}}}=\frac{v}{\Lambda} \frac{1-p}{1+p}
$$

其中 $p$ 为描述边界粗糙程度的参数, $\Lambda$ 为描述材料横 截面积的参数.

本文主要考虑边界散射对热导率的影响, 可近 似认为在低温状态下, $\tau_{\mathrm{to}} \approx \tau_{\mathrm{b}}$. 并将公式(13)转化为相 对于波数 $q$ 的积分形式

$$
\kappa \cong \frac{\hbar^{2} \Lambda}{k_{\mathrm{B}} V T^{2}} \frac{1+p}{1-p} \sum_{s} \int_{0}^{q_{m}} v \omega^{2} \frac{\mathrm{e}^{x}}{\left(\mathrm{e}^{x}-1\right)^{2}} \mathrm{~d} q
$$

其中 $x=\hbar \omega / k_{\mathrm{B}} T$. 当考虑线性色散关系时, 可近似认 为 $\omega \approx v \cdot q$, 并且在低温段, $x \rightarrow \infty$, 公式 (15) 可表示 为

$$
\kappa \approx \frac{\Lambda T}{V} \frac{1+p}{1-p} \sum_{s} \int_{0}^{\infty} x^{2} \frac{\mathrm{e}^{x}}{\left(\mathrm{e}^{x}-1\right)^{2}} \mathrm{~d} x
$$

所以在低温段纳米管的热导率与温度符合 $\kappa \propto T$ 关 系.

\section{3 尺寸效应}

根据声子散射理论, 模型轴向长度的变化将因 为其逐渐接近或者远离声子平均自由程而对声子 在热输运过程中的散射产生影响, 进而影响模型的 热输运性能. 本文建立了长度为 10-120 $\mathrm{nm}$ 的 10 10 扶手椅型 BNNT, 模拟温度选择为 $300 \mathrm{~K}$, 获得结 果如图 9 所示.

如图 9 可知, BNNT 的轴向热导率随着轴向长 度的增加而增加, 且增加的速率逐渐减小.

根据声子散射理论, 随着 BNNT 轴向长度的增 加, 声子在传输过程中与边界的碰撞概率逐渐减 小, 这将增加声子平均自由程, 根据公式(6), BNNT 的热输运性能逐渐增加; 随着 BNNT 轴向长度逐渐

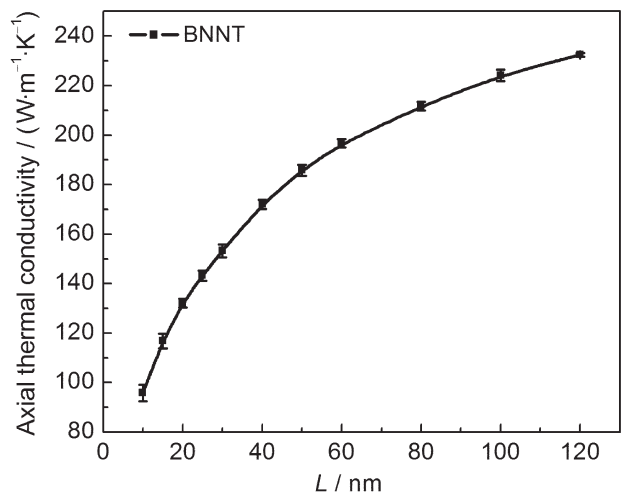

图9BNNT轴向热输运性能与长度的关系

Fig.9 Relationship between axial thermal conductivity and length
接近声子平均自由程, 声子间相互碰撞概率逐渐增 大, 这对随着长度增加而逐渐减小的声子与边界的 碰撞概率起到一个抵消作用, 根据上文分析, BNNT 的热输运性能增加趋势逐渐减缓. Mingo 和 Broido ${ }^{14}$ 通过计算 $\mathrm{CNT}$ 的 BTE 得到热导率与长度呈 $\kappa \propto L^{a}$ 这一关系, 且随着 $L$ 的增加, $\alpha$ 值(描述模型弹道特性 的参数)逐渐减小. 本文通过 MD 获得的结果如拟合 为 $\kappa \propto L^{\alpha}$ 形式, 其 $\alpha$ 值大致介于 0.1989-0.4899 之间, 且随着长度的增加, $\alpha$ 值逐渐减小, 这与 Mingo 的理 论结果相吻合. 同时这也意味着随着 BNNT 长度的 增加, 模型的弹道性能逐渐减弱, 但其仍主要体现 弹道特征.

\section{4 结 论}

运用 NEMD研究了 BNNT 的热输运性能, 分别 研究了长度、温度和应变对 BNNT 的热输运性能的 影响, 并得到了以下结论: 在拉伸应变作用下, 随着 应变的增加, 受声子模式变化的影响, BNNT 的热导 率呈逐渐降低的趋势; 在压缩应变作用下, 随着应 变的增加, 受 BNNT 发生显著塑性变形的影响, 其 热导率逐渐减小. 当外界温度低于 $70 \mathrm{~K}$ 时, 受量子 效应的影响, BNNT 的热导率随着温度的增加呈线 性增加趋势; 当温度高于 $100 \mathrm{~K}$ 时, 其热导率随着温 度的增加而逐渐减小. 当 $\mathrm{BNNT}$ 轴向长度小于 120 $\mathrm{nm}$ 时, 随着长度的增加, 其弹道性能逐渐减弱, 但仍 主要体现为弹道特征, 其热导率与长度基本满足 $\kappa \propto L^{\alpha}$ 这一关系

\section{References}

(1) Iijima, S. Nature 1991, 354, 56

(2) Rubio, A.; Corkill, J. L.; Cohen, M. L. Phys. Rev. B 1994, 49 (7), 5081.

(3) Loiseau, A.; Willaime, F.; Demoncy, N.; Hug, G.; Pascard, H. Phys. Rev. Lett. 1996, 76 (25), 4737.

(4) Wirtz, L.; Rubio, A. Phys. Rev. B 2003, 68 (4), 045425.

(5) Zhang, G.; Li, B. W. The Journal of Physical Chemistry B 2005, $109(50), 23823$

(6) Liew, K. M.; Yuan, J. H. Nanotechnology 2011, 22, 085701

(7) Xiao, Y.; Yan, H.; Cao, J. X.; Ding, J. W.; Mao, Y. L.; Xiang, J. Phys. Rev. B 2004, 69 (20), 205415.

(8) Chang, C. W.; Fennimore, A. M.; Afanasiev, A.; Okawa, D.; Ikuno, T.; Garcia, H.; Li, D. Y.; Majumdar, A.; Zettl, A. Phys. Rev. Lett. 2006, 97, 085901.

(9) Chang, C. W.; Okawa, D.; Majumdar, A.; Zettl, A. Science 2006, 314, 1112 . 
(10) Zhi, C. Y.; Bando, Y.; Tang, C. C.; Golberg, D. Materials Science and Engineering $R$ 2010, 70, 92.

(11) Barman, S. Europhysics Letters 2011, 96, 16004.

(12) Stewart, D. A.; Savic, N.; Mingo, N. Nano Lett. 2009, 9, 81.

(13) Cho, H. B.; Tokoi, Y.; Tanaka, S.; Suematsu, H.; Suzuki, T.; Jiang, W. H.; Niihara, K.; Nakayama, T. Composites Science and Technology 2011, 71, 1046.

(14) Mingo, N.; Broido, D. A. Nano Lett. 2005, 5 (7), 1221.

(15) Chang, C. W.; Han, W. Q.; Zettl, A. Journal of Vacuum Science \& Technology B 2005, 23, 1883.

(16) Tang, C. C.; Bando, Y.; Liu, C. H.; Fan, S. S.; Zhang, J.; Ding, X. X.; Golberg, D. The Journal of Physical Chemistry B 2006, $110(21), 10354$.

(17) Tersoff, J. Phys. Rev. B 1988, 37 (12), 6991.

(18) Wang, X. W.; Huang, Z.; Wang, T.; Tang, Y. W.; Zeng, X. C. Physica B 2008, 403, 2021.

(19) Sandia National Laboratories. LAMMPS, Lammps-12Oct10; GNU: USA, 2010.

(20) Schelling, P. K.; Phillpot, S. R.; Keblinski, P. Phys. Rev. B 2002, 65 (14), 144306.

(21) Lindsay, L.; Broido, D. A.; Mingo, N. Phys. Rev. B 2009, 80, 125407.

(22) Wang, S. C.; Liang, X. G.; Xu, X. H.; Ohara, T. J. Appl. Phys. 2009, $105,014316$.

(23) Turney, J. E.; McGaughey, A. J. H.; Amon, C. H. Phys. Rev. B
2009, 79 (22), 224305.

(24) Munoz, E.; Lu, J. X.; Yakobson, B. I. Nano Lett. 2010, 10 (5), 1652.

(25) Hu, J. N.; Ruan, X. L.; Chen, Y. P. Nano Lett. 2009, 9 (7), 2730.

(26) Sese L. M. Mol. Phys. 1993, 78,1167.

(27) Tchouar, N.; Ould, K. F.; Levesque, D. J. Chem. Phys. 2004, $121,7326$.

(28) Goharshadi, E. K.; Abbaspour, M.; Kashani, H.; Baherololoom, M. Theor. Chem. Acc. 2008, 119, 355.

(29) Yang, N.; Zhang, G.; Li, B. W. Nano Lett. 2008, 8 (1), 276.

(30) Kang, D. D.; Hou, Y.; Dai, J. Y.; Yuan, J. M. Phys. Rev. A 2009, 79, 063202 .

(31) Huang, M. J.; Weng, C. C.; Chang, T. M. International Journal of Thermal Sciences 2009, 49, 1095.

(32) Ren, C. L.; Zhang, W.; Xu, Z. J.; Zhu, Z. Y.; Huai, P. The Journal of Physical Chemistry C 2009, 114 (13), 5786.

(33) Xu, Z. P.; Buehler, M. J. Nanotechnology 2009, 20, 185701.

(34) Shen, H. J. Computational Materials Science 2009, 47, 220.

(35) Jiang, J. W.; Wang, J. S. Phys. Rev. B 2011, 84 (8), 085439.

(36) Goicochea, J. V.; Madrid, M.; Amon, C. ASME J. Heat Transfer 2010, 132, 012401 .

(37) Chen, Y. F.; Li, D. Y.; Lukes, J. R.; Majumdar, A. ASME J. Heat Transfer 2005, 127, 1129.

(38) Nika, D. L.; Pokatilov, E. P.; Askerov, A. S.; Balandin, A. A. Phys. Rev. B 2009, 79 (15), 155413. 